\title{
Maximum planar subgraphs in dense graphs
}

\author{
Peter Allen \\ Department of Mathematics \\ London School of Economics \\ Houghton Street, London WC2A 2AE, United Kingdom \\ p.d.allen@lse.ac.uk \\ Jozef Skokan \\ Department of Mathematics \\ London School of Economics \\ Houghton Street, London WC2A 2AE, United Kingdom \\ and \\ Department of Mathematics \\ University of Illinois \\ 1409 W. Green Street, Urbana, IL 61801, United States \\ j.skokan@lse.ac.uk \\ Andreas Würfl \\ Zentrum Mathematik \\ Technische Universität München \\ Boltzmannstrasse 3 D-85748, Garching bei München, Germany \\ wuerfl@ma.tum.de
}

Submitted: Jan 8, 2013; Accepted: June 28, 2013; Published: July 19, 2013

Mathematics Subject Classifications: 05C35, 05C10

\begin{abstract}
Kühn, Osthus and Taraz showed that for each $\gamma>0$ there exists $C$ such that any $n$-vertex graph with minimum degree $\gamma n$ contains a planar subgraph with at least $2 n-C$ edges. We find the optimum value of $C$ for all $\gamma<1 / 2$ and sufficiently large $n$.
\end{abstract}

\section{Introduction}

A way to reformulate typical questions in extremal graph theory is the following. Given a property $\mathcal{P}$ and an edge density (or minimum vertex degree, etc.), what is the 'largest' 
member of $\mathcal{P}$ which must be contained in an $n$-vertex graph $G$ with the given density? For many problems in extremal graph theory, the property $\mathcal{P}$ is somewhat trivial (for example, in Turán's theorem, $\mathcal{P}$ is the set of cliques). However this is not always the case: for example, in the Erdős-Stone [4] theorem, $\mathcal{P}$ is the set of complete $r$-partite graphs, and the problem of determining the 'largest' complete $r$-partite subgraph remains active, with most recently results and generalisations due to Nikiforov [10]. In 2005, Kühn, Osthus and Taraz [9] suggested the study of the property $\mathcal{P}$ consisting of all planar graphs, which, while well-studied in other parts of graph theory, have received relatively little attention from extremal graph theorists.

A plane graph is a drawing of a graph in the plane with no crossing edges. A graph is called planar if it has a plane graph drawing. The planarity of a graph $G$ is defined as the maximum number of edges in a planar subgraph of $G$. We denote the planarity of $G$ by $\operatorname{pl}(G)$. Kühn, Osthus and Taraz [9] investigated the connection between the minimum degree $\delta(G)$ and planarity $\operatorname{pl}(G)$ of a graph $G$ by studying the parameter

$$
\operatorname{pl}(n, d):=\min \{\operatorname{pl}(G):|G|=n, \delta(G) \geqslant d\} .
$$

Among other results they proved the following theorem.

Theorem 1. For each $\gamma>0$ there exists a constant $n_{\gamma}$ such that $\mathrm{pl}(n,(2 / 3+\gamma) n)=3 n-6$ for every integer $n \geqslant n_{\gamma}$.

This was later improved by Kühn and Osthus [8] to the following result with the optimal bound on the minimum degree.

Theorem 2. There exists $n_{2}$ such that $\operatorname{pl}(n, 2 n / 3)=3 n-6$ for every integer $n \geqslant n_{2}$.

More recently, Cooley, Łuczak, Taraz and Würfl [3] showed the following threshold behaviour of $\operatorname{pl}(n, d)$ at minimum degree $d=n / 2$.

Theorem 3. For every $\mu>0$ there exists $n_{\mu}$ such that, for every $n \geqslant n_{\mu}$, we have that

$$
\operatorname{pl}(n,\lceil n / 2\rceil) \geqslant(2.25-\mu) n \quad \text { for } n \text { odd, }
$$

and

$$
\operatorname{pl}(n, n / 2+1) \geqslant(2.5-\mu) n \quad \text { for } n \text { even } .
$$

This indeed constitutes a threshold behaviour since $\operatorname{pl}(n,\lfloor n / 2\rfloor) \leqslant 2 n-4$ for all integers $n$ as one can see from the class of complete bipartite graphs. For smaller values of $d$ one does not observe such rapid changes in the planarity. Indeed, Kühn, Osthus and Taraz [9] showed that $\mathrm{pl}(n, d)$ varies only by a constant term for the whole range of $d=\gamma n$ with $\gamma \in(0,1 / 2)$.

Theorem 4. For each $\gamma>0$ there is $C=C(\gamma)$ such that $\operatorname{pl}(n, \gamma n) \geqslant 2 n-C$ for every integer $n$. 
For $\gamma<1 / 2$ this is optimal up to the value of the constant $C$. For $\gamma \geqslant 1 / 2$ the above statement trivially holds: a Hamilton cycle with chords from one vertex on the inner face and from another vertex on the outer face proves that every $n$-vertex graph with minimum degree at least $n / 2$ has a planar subgraph with $2 n-4$ edges. So it is natural to ask whether there are values $\gamma<1 / 2$ such that $C(\gamma)=4$. We answer this in the affirmative as we determine the optimal value of $C(\gamma)$ for all $0<\gamma<1 / 2$.

Theorem 5. For every $\gamma \in(0,1 / 2)$ there exists $n_{\gamma}$ such that $\mathrm{pl}(n, \gamma n)=2 n-4 k$ for every $n \geqslant n_{\gamma}$, where $k \in \mathbb{N}$ is the unique integer such that $k \leqslant 1 /(2 \gamma)<k+1$. Hence, $C(\gamma)=4\lfloor 1 /(2 \gamma)\rfloor$ for $n \geqslant n_{\gamma}$.

Note that the constants are best possible for the given minimum degree condition: the graph consisting of $k$ disjoint copies of $K_{t, t}$ has $2 k t$ vertices, is $t$-regular, and has no planar subgraph with more than $4 k t-4 k$ edges because $K_{t, t}$ has no planar subgraph with more than $4 t-4$ edges.

\section{Tools and lemmas}

Our main tools in the proof are variants of the Regularity Lemma [11] and the Blow-up Lemma [6]. In order to formulate the versions that we will use, we first introduce some terminology.

Let $G=(V, E)$ be a graph and let $\varepsilon, d \in(0,1]$. For disjoint nonempty sets $U, W \subseteq V$, we denote by $e(U, W)$ the number of edges between $U$ and $W$, and define the density of the pair $(U, W)$ as $d(U, W):=e(U, W) /|U||W|$. A pair $(U, W)$ is $\varepsilon$-regular if

$$
\left|d\left(U^{\prime}, W^{\prime}\right)-d(U, W)\right| \leqslant \varepsilon
$$

for all $U^{\prime} \subseteq U$ and $W^{\prime} \subseteq W$ with $\left|U^{\prime}\right| \geqslant \varepsilon|U|$ and $\left|W^{\prime}\right| \geqslant \varepsilon|W|$. If the pair $(U, W)$ is $\varepsilon$-regular and has density at least $d$, then we say that $(U, W)$ is $(\varepsilon, d)$-regular.

An $\varepsilon$-regular partition of $G=(V, E)$ is a partition $V_{0} \cup V_{1} \cup \ldots \cup V_{r}$ of $V$ with $\left|V_{0}\right| \leqslant$ $\varepsilon|V|,\left|V_{i}\right|=\left|V_{j}\right|$ for all $i, j \in[r]:=\{1, \ldots, r\}$, and such that, for all but at most $\varepsilon r^{2}$ pairs $(i, j) \in[r]^{2}$, the pair $\left(V_{i}, V_{j}\right)$ is $\varepsilon$-regular.

We say that an $\varepsilon$-regular partition $V_{0} \cup V_{1} \cup \ldots \cup V_{r}$ of a graph $G$ is an $(\varepsilon, d)$-regular partition if the following is true. For every $i \in[r]$ and every vertex $v \in V_{i}$, there are at most $(\varepsilon+d) n$ edges incident to $v$ which are not contained in $(\varepsilon, d)$-regular pairs of the partition.

Given an $(\varepsilon, d)$-regular partition $V_{0} \cup V_{1} \cup \ldots \cup V_{r}$ of a graph $G$, we define a graph $R$, called the reduced graph of the partition of $G$, where $R=(V(R), E(R))$ has $V(R)=$ $\left\{V_{1}, \ldots, V_{r}\right\}$ and $V_{i} V_{j} \in E(R)$ whenever $\left(V_{i}, V_{j}\right)$ is an $(\varepsilon, d)$-regular pair. We will usually omit the partition, and simply say that $G$ has $(\varepsilon, d)$-reduced graph $R$. We call the partition classes $V_{i}$ with $i \in[r]$ clusters of $G$. Observe that our definition of the reduced graph $R$ implies that, for $T \subseteq V(R)$, we can, for example, refer to the set $\bigcup T$, which is a subset of $V(G)$.

In our proof, we require the minimum degree form of the Regularity Lemma. 
Lemma 6 (Regularity Lemma, minimum degree form). For all positive $\varepsilon, d$ and $\gamma$ with $0<\varepsilon<d<\gamma<1$ there is $r_{1}$ such that every graph $G$ on $n>r_{1}$ vertices with minimum degree $\delta(G) \geqslant \gamma n$ has an $(\varepsilon, d)$-reduced graph $R$ on $r$ vertices such that $r \leqslant r_{1}$ and $\delta(R) \geqslant(\gamma-d-\varepsilon) r$.

Lemma 6 is an easy consequence of the original Regularity Lemma of Szemerédi [11]. Its proof can be found, for example, in [9, Proposition 9].

Now we outline our proof strategy for Theorem 5. First, we apply Lemma 6 to a given $n$-vertex graph $G$ with minimum degree at least $\gamma n$ and obtain the reduced $r$-vertex graph $R$ whose minimum degree is almost as large as $\gamma r$. Then we need to distinguish two cases.

Case 1: Some component of $R$ has less than $2 \delta(R)$ vertices, and therefore contains a triangle. Using this triangle we will find a small triangulation $T$ (that is, a plane graph whose every face is a triangle) in $G$, and Theorem 4 will guarantee a subgraph $S$ of the rest of the graph $G-V(T)$ such that the disjoint union of $S$ and $T$ has at least $2 n$ edges.

Case 2: Each component of $R$ has at least $2 \delta(R)>r /(k+1)$ vertices, and thus $R$ has at most $k$ components. These components correspond to $k$ well-connected subgraphs of $G$ and cover almost all vertices of $G$. In each subgraph we will find a quadrangulation (a plane graph whose every face has four edges) which has a certain 'accepting' property that allows the few remaining vertices to be inserted. We conclude that there is a collection of at most $k$ vertex-disjoint quadrangulations covering all the vertices of $G$. Since every quadrangulation on $m$ vertices has $2 m-4$ edges, the theorem follows.

As one can see from the above outline, our argument divides into two cases, depending on whether the reduced graph $R$ has a small component or not. In each case we shall need some embedding results, which we now describe in detail.

When the reduced graph $R$ does have a small component, we will need the following embedding result, an easy case of the Counting Lemma (see, for example, Theorem 2.1 in [7]).

Lemma 7. For each $d>0$ and $s \in \mathbb{N}$ there exist $\varepsilon>0$ and $m_{0}$ such that whenever $m \geqslant m_{0}$ the following holds. Let $U, V, W$ be three pairwise disjoint vertex sets each of size $m$. Suppose that each pair forms an $(\varepsilon, d)$-regular pair in a graph $G$. Then $G$ contains every 3-partite triangulation on s vertices.

In the case that $R$ has no small components, we will construct quadrangulations. For this we shall use a version of the Blow-up Lemma. In order to state this result, we need a further definition. A pair of disjoint sets of vertices $U$ and $W$ in a graph $G$ is called $(\varepsilon, \delta)$-super-regular if it is $\varepsilon$-regular, each vertex $u \in U$ has at least $\delta|W|$ neighbours in $W$, and each $w \in W$ has at least $\delta|U|$ neighbours in $U$.

The original version of the Blow-up Lemma, due to Komlós, Sárközy and Szemerédi [6], showed that, for the purposes of embedding graphs of bounded degree, super-regular pairs behave like complete bipartite graphs. In our proof, we will need to embed (planar) graphs with growing degrees, which is generally a very difficult problem. Fortunately for us, planar graphs are examples of arrangeable graphs, for which a suitable extension of the Blow-up Lemma [6] has recently been proven by Böttcher, Kohayakawa, Taraz and Würfl. 
Definition 8 (a-arrangeable). Let a be an integer. An n-vertex graph is called a-arrangeable if its vertices can be ordered as $\left(x_{1}, \ldots, x_{n}\right)$ in such a way that

$$
\left|N\left(N\left(x_{i}\right) \cap\left\{x_{i+1}, x_{i+2}, \ldots, x_{n}\right\}\right) \cap\left\{x_{1}, x_{2}, \ldots, x_{i}\right\}\right| \leqslant a
$$

for each $1 \leqslant i \leqslant n$.

Here, for a set of vertices $S$, we denote by $N(S)$ the set of those vertices not in $S$ that are adjacent to some vertex in $S$.

Chen and Schelp showed that planar graphs are 761-arrangeable [2]; Kierstead and Trotter [5] improved this to 10-arrangeable. Thus, the following theorem of Böttcher, Kohayakawa, Taraz and Würfl [1] can be used to embed planar graphs whose maximum degree is not too large.

Theorem 9 (Arrangeable Blow-up Lemma). For all $a, \Delta_{R}, \kappa \in \mathbb{N}$ and for all $\delta>0$ there exists $\varepsilon>0$ such that for every integer $r$ there is $n_{0}$ such that the following is true for every $n_{1}, \ldots, n_{r}$ with $n_{0} \leqslant n=\sum n_{i}$ and $n_{i} \leqslant \kappa \cdot n_{j}$ for all $i, j \in[r]$.

Let $R$ be a graph of order $r$ with $\Delta(R)<\Delta_{R}$. Assume that we are given a graph $G$ with a partition $V(G)=V_{1} \cup \ldots \cup V_{r}$ and a graph $H$ with a partition $V(H)=X_{1} \cup \ldots \cup X_{r}$ with $\left|V_{i}\right|=\left|X_{i}\right|=n_{i}$ such that $\left(V_{i}, V_{j}\right)$ is an $(\varepsilon, \delta)$-super-regular pair for every $i j \in E(R)$ and such that all edges of $H$ run between sets $X_{i}, X_{j}$ for which $i j \in E(R)$. Further assume that $H$ is a-arrangeable and has $\Delta(H) \leqslant \sqrt{n} / \log n$. Then there exists an embedding $\varphi: V(H) \rightarrow V(G)$ of $H$ to $G$ such that $\varphi\left(X_{i}\right)=V_{i}$.

We will embed planar graphs into large well connected subgraphs of $G$. These subgraphs will correspond to spanning trees of components of $R$. However, to be able to use Theorem 9, we shall need spanning trees whose maximum degree is bounded. This is the purpose of the following lemma.

Lemma 10. Given $k \in \mathbb{N}$, let $R$ be a connected graph with minimum degree at least $v(R) / k$. Then $R$ has a spanning tree with maximum degree $4 k$.

Proof. We define the score of a spanning tree $T$ of $R$ to be the sum of the squares of the degrees of vertices in $T$. Let $T$ be a spanning tree of $R$ with minimum score. Observe that $T$ has less than $v(R) /(2 k)$ vertices of degree $4 k$, since the sum of the vertex degrees of $T$ is $2 v(R)-2$.

Suppose that there is a vertex $u$ of $T$ whose degree in $T$ exceeds $4 k$. Observe that the removal of $u$ from $T$ disconnects $T$ into more than $4 k$ components, one of which, $C$, has less than $v(R) /(4 k)$ vertices. Let $v$ be the neighbour of $u$ which is in $C$. Now $v$ has at least $v(R) / k$ neighbours in $R$, of which less than $v(R) /(4 k)$ are in $C$ and a further less than $v(R) /(2 k)$ are of degree at least $4 k$. It follows that $v$ has a neighbour $u^{\prime}$ in $R$ which is not in $C$ and whose degree is less than $4 k$. Let $T^{\prime}$ be obtained from $T$ by deleting $u v$ and inserting $u^{\prime} v$. Then $T^{\prime}$ is still a spanning tree of $v$. Each vertex of $T^{\prime}$ has the same degree as in $T$ except for $u$ and $u^{\prime}$, which have respectively lost and gained one neighbour. It follows that the score of $T^{\prime}$ is smaller than that of $T$, which by contradiction completes the proof. 
Finally, we need to specify which planar graphs we will embed into our spanning trees.

Let $H=(V, E)$ be a plane graph. We say that a $k$ element subset $V^{\prime} \subseteq V$ forms a bag of order $k$ in $H$ if there is $\left\{x_{1}, x_{2}\right\} \subseteq V \backslash V^{\prime}$ such that $V^{\prime} \cup\left\{x_{1}, x_{2}\right\}$ induces a copy of $K_{2, k}$ in $H$ and all inner faces of $H\left[V^{\prime} \cup\left\{x_{1}, x_{2}\right\}\right]$ are also faces of $H$. We call the vertices of $V^{\prime}$ which are not in the outer face the interior vertices of the bag.
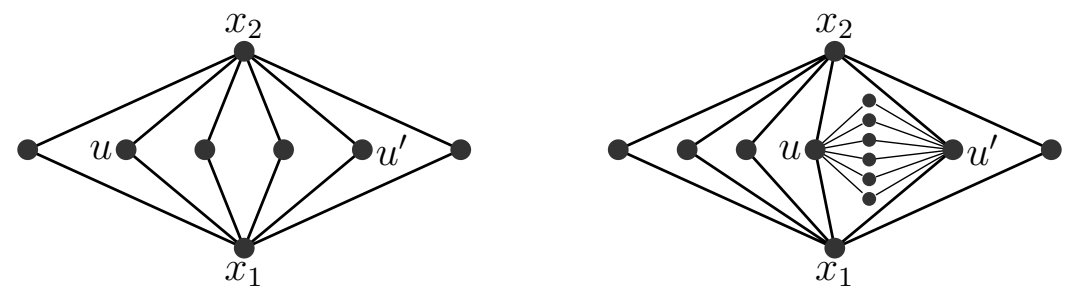

Figure 1: A bag of order $k=6$; the same bag reordered and with an insertion of $\ell=6$ vertices.

Observe that we can reorder the interior vertices of a bag without affecting the planarity of $H$. A bag is thus a very convenient structure into which one can put further vertices: if some vertex $v$ (not in $H$ ) is adjacent in a supergraph $G$ of $H$ to any two interior vertices $u, u^{\prime}$ of a bag, then we can redraw $H$ such that $u$ and $u^{\prime}$ are consecutive in the bag, and insert $v$ and $u v, u^{\prime} v$ to obtain $H^{\prime}$. If $H$ is a quadrangulation, then $H^{\prime}$ is still a quadrangulation contained in $G$. Furthermore, if $v_{1}, \ldots, v_{\ell} \notin V(H)$ are all adjacent to $u$ and $u^{\prime}$ in $G$, then we can insert all these vertices and edges to $u$ and $u^{\prime}$, and still obtain a subgraph $H^{\prime \prime}$ of $G$ which is a quadrangulation. Furthermore, $v_{1}, \ldots, v_{\ell}$ then form a bag of order $\ell$ in $H^{\prime \prime}$. This will be particularly useful in the proof of the following lemma.

Lemma 11. Let $T$ be a tree of order $r \geqslant 2, n \geqslant(16 r)^{3}$, and let $G$ be an n-vertex graph with the partition $V_{1} \cup \ldots \cup V_{r}$ of its vertex set such that $\left|V_{i}\right| \leqslant 2\left|V_{j}\right|$ for all $i \neq j$ and $G\left[V_{i}, V_{j}\right]$ is a complete bipartite graph whenever $i j \in E(T)$.

Then $G$ contains a plane quadrangulation $H$ with maximum degree $\Delta(H) \leqslant n^{1 / 3}+2$ as a spanning subgraph. Furthermore, all but at most $9 n^{2 / 3}$ vertices of $H$ are contained in a collection of pairwise disjoint bags each of order in the interval $\left[n^{1 / 3} / 2, n^{1 / 3}\right]$.

Proof. We first prove that $G$ has a quadrangulation $H$ with $\Delta(H) \leqslant n^{1 / 3}+2$ by induction on $r$. So assume that $r=2$ and $G$ is a bipartite graph with partite sets $V_{1}, V_{2}$. We partition $V_{i}$ into a minimum number of sets $W_{i, j}$ with sizes $\left|W_{i, j}\right| \leqslant n^{1 / 3}$ as equal as possible. The plane graph $H$ is constructed as follows. Take $x_{1}, x_{2} \in W_{1,1}$ and all of $W_{2,1}$ and embed the graph induced by these vertices into the plane. Let $y_{1}, y_{2} \in W_{2,1}$ lie in the same face and embed $W_{1,1} \backslash\left\{x_{1}, x_{2}\right\}$ into this face connecting each vertex to $y_{1}$ and $y_{2}$. We continue greedily embedding sets $W_{i, j}$ into faces with two vertices of degree 2 from $V_{3-i}$ and adding all edges in between. This process does not stop before all vertices of $G$ have been embedded into the plane. The resulting graph $H$ is a quadrangulation with $\Delta(H) \leqslant n^{1 / 3}+2$. 
Now assume that $r>2$ and $1 r \in E(T)$. Further assume that we have embedded $V_{1} \cup \ldots \cup V_{r-1}$ this way and obtained a quadrangulation $H^{\prime}$ on $V \backslash V_{r}$. We extend $H^{\prime}$ to a quadrangulation $H$ on $V$ as follows. Again partition $V_{r}$ into a minimum number of sets $W_{r, i}$ with sizes $\left|W_{r, i}\right| \leqslant n^{1 / 3}$ as equal as possible. For each $i$ sequentially, pick a pair of vertices $u, u^{\prime}$ in $V_{1}$ that have degree 2 and lie in the same face in $H^{\prime}$, embed all vertices from $W_{r, i}$ into this face, and connect these to $u, u^{\prime}$. Since $\left|V_{1}\right| \geqslant n /(2 r-1)>8 n^{2 / 3}$, we do not run out of pairs in $V_{1}$.

It remains to show that most vertices lie in a collection of large disjoint bags. Recall that the planarity of $H$ is preserved if we reorder the embedding in such a way that all vertices in $W_{i, j}$ of degree 2 in $H$ form a bag. Since there are at most $2 n^{2 / 3}$ many sets $W_{i, j}$, all but at most $4 n^{2 / 3}$ many vertices lie in pairwise disjoint bags. Some of these bags might be small, i.e., they might have order less than $\frac{1}{2} n^{1 / 3}$. Assume that the bag in $W_{i, j}$ is small. Note that $\left|W_{i, j}\right| \geqslant \frac{9}{10} n^{1 / 3}$ by construction. Thus at least $\frac{1}{5} n^{1 / 3}$ pairs from $W_{i, j}$ have been used to embed other sets $W_{i^{\prime}, j^{\prime}}$. But there are at most $2 n^{2 / 3}$ many sets $W_{i, j}$. Hence, at

most $10 n^{1 / 3}$ bags are small. Consequently, all but at most $4 n^{2 / 3}+10 n^{1 / 3} \cdot \frac{1}{2} n^{1 / 3} \leqslant 9 n^{2 / 3}$ vertices lie in disjoint bags of size at least $\frac{1}{2} n^{1 / 3}$.

\section{Proof of Theorem 5}

Given $\gamma>0$, let $k \in \mathbb{N}$ be such that $k \leqslant 1 /(2 \gamma)<k+1$. We set $\beta=\gamma-1 /(2(k+1))$, $\delta=\beta / 8, d=\beta / 4$ and $s=C(\beta)+6$, where $C(\beta)$ is the constant returned by Theorem 4 . Next we choose $\varepsilon$ such that $2 \varepsilon$ is sufficiently small to apply Theorem 9 with $a=10$, $\Delta_{R}=8(k+1)+1, \kappa=2$ and $\delta$ as given. We further insist that

$$
\varepsilon \leqslant \frac{\beta}{10^{5} k^{4}(8(k+1)+2)} .
$$

Let $r_{1}$ be the parameter returned by Lemma 6 for $d$ and $\varepsilon$ as chosen, and $m_{0}$ that returned by Lemma 7 . Let $n_{0} \geqslant \max \left\{\left(16 r_{1}\right)^{3}, 6(k+1) s, m_{0} r_{1}\right\}$ be sufficiently large so that Theorem 9 applies with any $r \leqslant r_{1}$.

Suppose that $n \geqslant(4 k) \cdot n_{0}$ and let $G$ be an $n$-vertex graph with minimum degree $\delta(G) \geqslant\left(\frac{1}{2(k+1)}+\beta\right) n$. By Lemma 6 there is an $(\varepsilon, d)$-regular partition $V(G)=V_{0} \bullet \ldots \cup V_{r}$ with $r \leqslant r_{1}$ such that the corresponding reduced graph $R$ satisfies $\delta(R) \geqslant\left(\frac{1}{2(k+1)}+\beta / 2\right) r$. We distinguish two cases.

Case 1: $R$ has a component with less than $2 \delta(R)$ vertices. In this case $R$ contains a triangle. It follows by Lemma 7 that $G$ contains a triangulation $T$ on $s$ vertices, which has $3 s-6$ edges. The graph $G-V(T)$ has minimum degree at least $\left(\frac{1}{2(k+1)}+\beta\right) n-s \geqslant \beta n$, where the last inequality is by our choice of $n_{0}$. Therefore, by Theorem $4, G-V(T)$ contains a planar subgraph $S$ with at least $2(n-s)-C(\beta)$ edges. Then $G$ contains the disjoint union of $S$ and $T$, which is planar and has at least $2 n-2 s-C(\beta)+3 s-6=2 n$ edges (by choice of $s$ ) as required.

Case 2: Every component of $R$ has at least $2 \delta(R)>r /(k+1)$ vertices. It follows that $R$ has $c \leqslant k$ components. We will show that we can cover $G$ with $c$ vertex-disjoint 
quadrangulations, which implies that $G$ contains a planar subgraph with at least $2 n-4 c \geqslant$ $2 n-4 k$ edges as required.

Let $C$ be a component of $R$, and $T$ be its spanning tree with maximum degree $8(k+1)$ guaranteed by Lemma 10. Let $V_{i}$ be any cluster of $C$ and $i j \in T$. Observe that, by the $(\varepsilon, d)$-regularity of $\left(V_{i}, V_{j}\right)$, at most $\varepsilon\left|V_{i}\right|$ vertices do not have at least $(d-\varepsilon)\left|V_{j}\right|$ neighbours in $V_{j}$. It follows that we can remove from each cluster $V_{i}$ at most $8(k+1) \varepsilon\left|V_{i}\right|$ vertices and obtain a set $V_{i}^{\prime}$ whose every vertex has at least $(d-\varepsilon)\left|V_{j}\right|$ neighbours in each $V_{j}$ such that $i j \in T$. Since $(8(k+1)+1) \varepsilon \leqslant \beta / 8$ and $d-\delta=\beta / 8$, if $i j \in T$, then each vertex in $V_{i}^{\prime}$ has at least $\delta\left|V_{j}^{\prime}\right|$ neighbours in $V_{j}^{\prime}$. Moreover, since $8(k+1) \varepsilon \leqslant 1 / 2$, for each $i$ we have $\left|V_{i}^{\prime}\right| \geqslant\left|V_{i}\right| / 2$. Consequently, the pair $\left(V_{i}^{\prime}, V_{j}^{\prime}\right)$ is $2 \varepsilon$-regular (see [7, Fact 1.5]) and, since $\left|V_{i}\right|=\left|V_{j}\right|$, we also have $\left|V_{i}^{\prime}\right| \leqslant 2\left|V_{j}^{\prime}\right|$ for each $i, j$. It follows that each edge $i j$ of $T$ corresponds to a $(2 \varepsilon, \delta)$-super-regular pair $\left(V_{i}^{\prime}, V_{j}^{\prime}\right)$, and the cluster sizes are not too unbalanced, as required for Theorem 9 .

Let $G^{\prime}$ be a graph whose vertex set is the union of the sets $V_{i}^{\prime}, i \in C$ and whose edges are all edges between $V_{i}^{\prime}$ and $V_{j}^{\prime}$ whenever $i j \in T$. Note that $G^{\prime}$ has $n_{C}$ vertices, where $n_{C}$ satisfies

$$
n \geqslant n_{C} \geqslant 2 \delta(R)(1-8(k+1) \varepsilon) \frac{(1-\varepsilon) n}{r} \geqslant \frac{n}{2 k} \geqslant n_{0} .
$$

By Lemma 11, $G^{\prime}$ contains a plane quadrangulation $H$ in which the maximum degree is at most $n_{C}{ }^{1 / 3}+2 \leqslant n^{1 / 3}+2$, and in which at most $9 n_{C}^{2 / 3}$ vertices are not contained in bags of order between $\frac{1}{2} n_{C}^{1 / 3} \geqslant(n / 16 k)^{1 / 3}$ and $n_{C}^{1 / 3} \leqslant n^{1 / 3}$. By Theorem $9, H$ can be embedded into the subgraph of $G$ induced on $\bigcup C$.

Repeating this for each component we obtain a collection of $c$ vertex disjoint quadrangulations $H_{1}, \ldots, H_{c}$ in $G$, together with a collection $B_{1}, \ldots, B_{\ell}$ of pairwise disjoint bags of order at least $(n / 16 k)^{1 / 3}$ covering all but at most $9 \sum_{C} n_{C}^{2 / 3} \leqslant 9 n^{2 / 3} k$ vertices of $\bigcup_{i \in[r]} V_{i}^{\prime}$. In particular, we have that $\frac{1}{2} n^{2 / 3}<\ell \leqslant(16 k)^{1 / 3} n^{2 / 3}$.

Let $L$ be the set of vertices in none of the quadrangulations. Observe that every vertex in $L$ is either in $V_{0}$ or in $V_{i} \backslash V_{i}^{\prime}$ for some $i$; therefore, it follows that $|L| \leqslant(8(k+1)+1) \varepsilon n$. We say that a bag $B_{i}$ is good for $u \in L$ if $u$ has at least $n^{1 / 3} /\left(32 k^{2}\right)$ neighbours in $B_{i}$. Since $(8(k+1)+1) \varepsilon n+9 n^{2 / 3} k \leqslant \beta n$, each vertex $u \in L$ has at least $n /(2(k+1))$ neighbours contained in $B_{1} \cup \cdots \cup B_{\ell}$. Of these at least $n /(2(k+1))-\ell \cdot n^{1 / 3} /\left(32 k^{2}\right) \geqslant n /(6 k)$ lie in bags that are good for $u$. Hence, at least $n^{2 / 3} /(6 k) \geqslant \ell /\left(24 k^{2}\right)$ of the bags $B_{i}$ are good for $u$. We now assign vertices $L_{i}$ of $L$ to each bag $B_{i}$ sequentially as follows. From the collection of unassigned vertices of $L$ for which $B_{i}$ is good, we assign $L_{i}$ to be any $n^{1 / 3} /\left(128 k^{2}\right)$ of them if this is possible, and all of them if not. Suppose that after carrying out this procedure there is a vertex $u$ of $L$ which is not in any $L_{i}$. Then it must be the case that for each $B_{i}$ good for $u$, we have $\left|L_{i}\right|=n^{1 / 3} /\left(128 k^{2}\right)$. But there are at least $\ell /\left(24 k^{2}\right)$ such $B_{i}$, and $\ell /\left(24 k^{2}\right) \cdot n^{1 / 3} /\left(128 k^{2}\right)>(8(k+1)+1) \varepsilon n \geqslant|L|$, (where the first inequality is by choice of $\varepsilon$ ) which is a contradiction.

We then work as follows. For each bag $B_{i}$, we reorder the interior vertices of $B_{i}$ such that the first vertex of $L_{i}$ is adjacent to the first and second interior vertices of $B_{i}$, the second vertex of $L_{i}$ to the third and fourth, and so on. Because each vertex of $L_{i}$ has at least $n^{1 / 3} /\left(32 k^{2}\right)$ neighbours in $B_{i}$, and $\left|L_{i}\right| \leqslant n^{1 / 3} /\left(128 k^{2}\right)$, this is possible. We now 
insert, for each $j$, the $j$ th vertex of $L_{i}$ into the interior face of $B_{i}$ containing the $(2 j-1)$ st and $2 j$ th interior vertices, and add the edges to those two vertices. Let the plane graphs so constructed be $H_{1}^{\prime}, \ldots, H_{c}^{\prime}$. By construction, these graphs are vertex disjoint and cover $G$, and since $H_{i}$ was a quadrangulation, so $H_{i}^{\prime}$ is also a quadrangulation for each $i$. The disjoint union of $H_{1}^{\prime}, \ldots, H_{c}^{\prime}$ is then a planar subgraph of $G$ with $2 n-4 c \geqslant 2 n-4 k$ edges, as required.

\section{Concluding Remarks}

There remain several open questions on planar graphs. In particular, it is possible that in Theorem 5 the constant $n_{\gamma}$ can be taken to be an absolute constant provided $\gamma \gg n^{-1 / 2}$. Note that this is a natural lower bound since there are bipartite graphs without 4-cycles of minimum degree $\Theta\left(n^{1 / 2}\right)$. Another possibility would be to investigate the behaviour of the planarity function $\mathrm{pl}(n, \gamma n)$ for $\gamma \in(1 / 2,2 / 3]$ in more detail. Finally, one could ask these questions if the constraint imposed is that of edge density rather than minimum degree.

More generally, one could replace 'planar graphs' by some other property — topologically defined, or by forbidden minors, for example.

\section{Acknowledgement}

The third author would like to thank the London School of Economics for their hospitality while this work was being completed.

\section{References}

[1] J. Böttcher, Y. Kohayakawa, A. Taraz, and A. Würfl, An extension of the Blow-up Lemma to arrangeable graphs, arXiv:1305.2059.

[2] G. Chen and R. H. Schelp, Graphs with linearly bounded Ramsey numbers, J. Combin. Theory Ser. B 57 (1993), no. 1, 138-149.

[3] O. Cooley, T. Łuczak, A. Taraz, and A. Würfl, Large planar subgraphs in dense graphs, in preparation, 2013.

[4] P. Erdös and A. H. Stone, On the structure of linear graphs, Bull. Amer. Math. Soc. 52 (1946), 1087-1091.

[5] H. A. Kierstead and W. T. Trotter, Planar graph coloring with an uncooperative partner, J. Graph Theory 18 (1994), no. 6, 569-584.

[6] J. Komlós, G. N. Sárközy, and E. Szemerédi, Blow-up Lemma, Combinatorica 17 (1997), no. 1, 109-123.

[7] J. Komlós and M. Simonovits, Szemerédi's regularity lemma and its applications in graph theory, Combinatorics, Paul Erdős is eighty, Vol. 2 (Keszthely, 1993), Bolyai Soc. Math. Stud., vol. 2, János Bolyai Math. Soc., Budapest, 1996, pp. 295-352. 
[8] D. Kühn and D. Osthus, Spanning triangulations in graphs., J. Graph Theory 49 (2005), no. 3, 205-233.

[9] D. Kühn, D. Osthus, and A. Taraz, Large planar subgraphs in dense graphs, J. Comb. Theory, Ser. B 95 (2005), no. 2, 263-282.

[10] V. Nikiforov, Some new results in extremal graph theory, arXiv:1107.1121.

[11] E. Szemerédi, Regular partitions of graphs, Problèmes combinatoires et théorie des graphes (Orsay, 1976), Colloques Internationaux CNRS, vol. 260, CNRS, 1978, pp. 399-401. 